\title{
Safety and efficacy of CAR-T cell targeting BCMA in patients with multiple myeloma coinfected with chronic hepatitis B virus
}

\author{
Lu Han, ${ }^{1}$ Jian Zhou, ${ }^{2}$ Keshu Zhou, ${ }^{2}$ Xinghu Zhu, ${ }^{2}$ Lingdi Zhao, ${ }^{1}$ Baijun Fang, ${ }^{2}$ \\ Qingsong Yin, ${ }^{2}$ Xudong Wei, ${ }^{2} \mathrm{Hu}$ Zhou, ${ }^{2}$ Linlin Li, ${ }^{1}$ Bengling Xu, ${ }^{1}$ Jishuai Zhang, ${ }^{3}$ \\ Yongping Song, ${ }^{2}$ Quanli Gao (i) ${ }^{1}$
}

To cite: Han L, Zhou J, Zhou K, et al. Safety and efficacy of CAR-T cell targeting BCMA in patients with multiple myeloma coinfected with chronic hepatitis B virus. Journal for ImmunoTherapy of Cancer 2020;8:e000927. doi:10.1136/ jitc-2020-000927

- Additional material is published online only. To view please visit the journal online (http://dx.doi.org/10.1136/jitc2020-000927).

$Q G$ and $Y S$ contributed equally.

Accepted 02 July 2020

Check for updates

(C) Author(s) (or their employer(s)) 2020. Re-use permitted under CC BY. Published by BMJ.

${ }^{1}$ Department of Immunology, Affiliated Cancer Hospital of Zhengzhou University and Henan Cancer Hospital, Zhengzhou,

China

${ }^{2}$ Department of Hematology, Affiliated Cancer Hospital of Zhengzhou University and Henan Cancer Hospital, Zhengzhou,

China

${ }^{3}$ The Shenzhen Pregene

Biopharma Company, Ltd,

Shenzhen, China

Correspondence to Dr Quanli Gao;

zlyygq10855@zzu.edu.cn

Professor Yongping Song; songyongping001@126.com

\section{ABSTRACT}

Background Reactivation of hepatitis B virus (HBV) infection is a well-recognized complication in patients with chronic or resolved HBV infection undergoing anticancer therapy. There is a risk of HBV reactivation after infusion of chimeric antigen receptor (CAR) T cells for patients with refractory/relapsed (R/R) multiple myeloma (MM).

Methods We administered $B$ cell maturation antigen (BCMA) CAR-T cell by infusion to nine patients with $\mathrm{R} / \mathrm{R}$ MM with chronic or resolved HBV infection. Patient serum was analyzed to determine the expression of five components of HBV and the copy number of HBV DNA. HBV reactivation was defined if a patient re-exhibited hepatitis B surface antigen (HBsAg) or HBV DNA regrowth after CAR-T therapy.

Results In one patient who was HBsAg-positive, no $\mathrm{HBV}$ reactivation was observed during the follow-up period of 9.8 months after administration of anti-HBV drugs before and after CAR-T therapy. Among eight patients with MM who had resolved HBV infection, two patients administered prophylactic anti-HBV drugs did not exhibit HBV reactivation. Of the six patients who did not use prophylactic antiviral drugs, five did not exhibit $\mathrm{HBV}$ reactivation, while one showed recurrence of $\mathrm{HBsAg}$ without detection of HBV DNA or damage to liver function. The best objective response rate was $100 \%$, and the progression-free survival (PFS) at 12 months was of 88.89\% (median PFS was not observed).

Conclusions These findings showed that BCMA CAR-T cell therapy could be used in patients with $R / R$ MM with chronic or resolved HBV infection and that antiviral drugs should be administered in these patients during CAR-T cell therapy.

\section{BACKGROUND}

Hepatitis B virus (HBV) infection is a serious health concern. Estimates have shown that more than 257 million people worldwide are infected with HBV and approximately 887,000 people die of $\mathrm{HBV}$ infection each year. ${ }^{1} \mathrm{HBV}$ reactivation is a well-recognized complication of anticancer therapy and can result in multiple clinical manifestations, ranging from asymptomatic hepatitis to fulminant hepatitis. ${ }^{23}$

HBV reactivation occurs in two separate clinical scenarios. First, patients with cancer who were hepatitis B surface antigen (HBsAg) positive (ie, patients with cancer with chronic HBV infection) can experience HBV reactivation, as demonstrated by an increase in serum HBV DNA levels and biochemical or clinical evidence of hepatitis. Second, patients who have a resolved HBV infection (HBsAg-negative with positive anti-hepatitis B core antibody (HBcAb), with or without an antibody to HBsAg (HBsAb)) can experience HBV reactivation. ${ }^{4-7}$ In patients with resolved HBV infection, a low level of HBV replication can be detected for many years in peripheral blood mononuclear cells and the liver, ${ }^{8}$ and HBV reactivation can occur during cytotoxic chemotherapy (CT), monoclonal antibody treatment, or bone marrow transplantation, with HBsAg appearance (HBsAg seroreversion). ${ }^{10-13}$

Chimeric antigen receptor (CAR)- $\mathrm{T}$ cell therapy is a novel treatment with definite curative effects for some hematological cancers. ${ }^{14-16} \mathrm{~B}$ cell maturation antigen (BCMA)-based CAR-T cells have significantly improved outcomes in patients with refractory/relapsed (R/R) multiple myeloma (MM), with objective response rates (ORRs) of more than $80 \%$ and complete remission (CR) rates of up to $60 \% .{ }^{17-20}$ To avoid viral reactivation or fulminant hepatitis, clinical trials of CAR-T cell therapy have generally excluded patients with aggressive MM coinfected with HBV. Thus, to date, the safety and efficacy of CAR-T cell therapy among patients with $\mathrm{MM}$ and $\mathrm{HBV}$ infection remain unexplored, and data on HBV reactivation in these patients are limited. Therefore, in this 


\begin{tabular}{|c|c|c|c|c|c|}
\hline 5'-LTR & CD8 $\alpha$ SP & $\begin{array}{l}V_{\mathrm{HH}} \text { binding } \\
\text { BCMA }\end{array}$ & $\begin{array}{c}\text { CD8 } \alpha \\
\text { Hinge+TM } \\
\end{array}$ & 4-1BB & $\mathrm{CD} 3$ \\
\hline
\end{tabular}

B

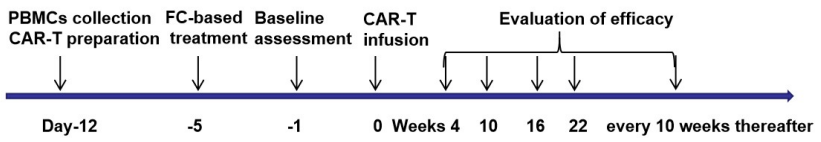

Figure 1 BCMA CAR-T cells and clinical treatment protocols. (A) Diagram of BCMA CAR, composed of a human CD8 $\alpha$ signal peptide (CD8 $\alpha \mathrm{sp}$ ), BCMA binding domain $\left(\mathrm{V}_{\mathrm{HH}}\right.$, variable domain of the heavy chain antibody), human CD8 $\alpha$ hinge and transmembrane domain (CD8 $\alpha$ hinge +TM), human 4-1BB cytoplasmic domain, and human CD3 $\zeta$

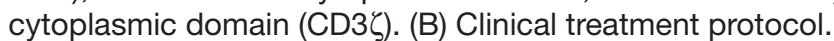
Patients underwent lymphocyte isolation to obtain peripheral blood lymphocytes on day -12 , and cells were transduced, cultured, and expanded. The first day of CAR-T infusion was established as study day 0 , and patients underwent fludarabine/cyclophosphamide-based lymphodepletion chemotherapy on day -5 . On day -1 ; weeks $4,10,16$, and 22; and every 10 weeks thereafter, efficacy was evaluated. $\triangle \mathrm{U} 3$, U3 region deletion; BCMA, B cell maturation antigen; CAR, chimeric antigen receptor; LTR, long terminal repeat; sp, signal peptide.

retrospective study, we evaluated the safety and efficacy of BCMA CAR-T cell therapy in patients with $\mathrm{R} / \mathrm{R} \mathrm{MM}$ and concomitant $\mathrm{HBV}$ infection.

\section{METHODS}

\section{Patient population}

This study was conducted at the Affiliated Cancer Hospital of Zhengzhou University and was registered with clinicaltrials.gov (registration number: NCT03664661). The study protocol was approved by the Ethics Committee of the Affiliated Cancer Hospital of Zhengzhou University. Informed consent was obtained from all patients.

Eligibility criteria were as follows: age, 18-70 years; Eastern Cooperative Oncology Group performance score, $\leq 2$ (on a scale of $0-5$, with higher scores indicating greater disability); measurable disease, based on serum monoclonal protein (M protein) $\geq 1.0 \mathrm{~g} / \mathrm{dL}$ in serum or $\geq 200 \mathrm{mg} / 24$ hours in urine, serum-free light chain (FLC) concentration of $\geq 10 \mathrm{mg} / \mathrm{dL}$ with an abnormal ratio, or bone marrow plasma cells $\geq 10 \%$; at least three previous lines of therapy, with each line of treatment having at least one complete treatment cycle; received an immunomodulatory drug (IMiD) and a proteasome inhibitor (PI), or had disease that was refractory to both drug classes; and adequate organ function. One additional criterion included BCMA expression in marrow plasma cells based on flow cytometry analysis. More details on the inclusion and exclusion criteria are given in the online supplementary appendix.

HBsAg, HBsAb, hepatitis B e antigen, hepatitis B e antibody (HBeAb), and $\mathrm{HBcAb}$ were detected by chemiluminescence by clinical laboratory analyzers using commercially available kits (Roche, Basel, Switzerland) before CAR-T cell therapy. HBV DNA was quantitated by PCR. Patients with active HBV infection prior to CAR-T cell therapy were excluded from the study. Patients who were HBsAg positive were given antiviral drugs during the entire treatment period, and prophylaxis with entecavir $(1 \mathrm{mg})$ or lamivudine $(100 \mathrm{mg})$ was recommended to patients who had resolved HBV infection until the immunoglobulin (Ig) levels recovered. As an indicator of HBV reactivation, hepatitis B serology and HBV DNA levels were closely monitored for all patients when evaluating efficacy during follow-up, and increasing testing number if there were obvious abnormal clinical hepatitis symptoms or the changes of liver function (bilirubin, transaminase, and albumin) after CAR-T cells infusion. HBV reactivation was indicated by (1) HBsAb absence and HBsAg appearance in patients who were HBsAg negative, with or without HBsAb; and (2) increase in HBV DNA levels by at least 10 -fold or $1 \times 10^{9}$ copies $/ \mathrm{mL} .{ }^{21}$ Antiviral treatment was initiated as soon as reactivation was detected. None of the patients had previously received hepatitis $\mathrm{B}$ vaccines.

\section{Preparation and administration of CAR-T cells}

BCMA CAR-T cells were manufactured according to Good Manufacturing Practices in the immunology laboratory of the Affiliated Cancer Hospital of Zhengzhou University. Cells expressing anti-BCMA CAR (figure 1A) were expanded over a period of 12 days, and CAR-T cells on day 12 were composed of a variable proportion of $\mathrm{CD} 4^{+}$ and $\mathrm{CD}^{+} \mathrm{T}$ cells by Fluorescence Activating Cell Sorter (FACS) analysis (online supplementary table 1). Patients underwent lymphodepletion with fludarabine and cyclophosphamide, followed by infusion of CAR-T cells on day 0 . On day -1 ; weeks $4,10,16$, and 22; and every 10 weeks thereafter, efficacy was evaluated (figure 1B). CAR DNA copy numbers were determined to evaluate CAR-T cells expansion and persistence. Additional information on this approach is provided in the online supplementary appendix.

\section{Evaluation of safety}

Adverse events were graded according to the National Cancer Institute's Common Terminology Criteria for Adverse Events (NCI CTCAE V.4.03), except for cytokinerelease syndrome (CRS). CRS was defined and graded according to published criteria proposed by Lee $e t a l^{22}$ Several serum biomarkers, including C-reactive protein (CRP), ferritin, and cytokines, were used to evaluate adverse events. Cytokines indicative of a response to the CAR-T cells were determined by ELISA kits (additional information is provided in the online supplementary appendix).

\section{Evaluation of clinical response}

The efficacy of the CAR-T cell therapy was evaluated according to the response criteria in the International Myeloma Working Group consensus recommendations. ${ }^{23}$ Patients were closely followed by bone marrow cytology or biopsy examination for plasma cells, minimal residual 
disease (MRD) of the bone marrow by flow cytometry, serum protein electrophoresis, immunofixation electrophoresis of serum and urine, serum FLC, CT scanning, and MRI.

\section{Statistical analysis}

The data were analyzed with GraphPad Prism V.6.0 and SPSS Statistics V.17.0. Data were presented as the mean \pm SD. Missing data were not imputed unless otherwise specified. Progression-free survival (PFS) and associated 95\% CIs were calculated using the Kaplan-Meier method.

\section{RESULTS \\ Clinical and hematological data for the patient cohort}

Between July 11, 2018, and April 17, 2019, nine patients with $\mathrm{R} / \mathrm{R}$ MM were enrolled in this study. The clinical and hematological characteristics are summarized in table 1 . According to the M-protein determination, the Ig or light-chain (LC) subtypes were $\operatorname{IgG} \lambda$ in three cases; IgG $\kappa$ in two cases; $\operatorname{IgA} \kappa$ in one case; $\operatorname{IgA} \lambda$ in one case; and $\kappa \mathrm{LC}$ in two cases. Five out of nine patients had received at least three lines of prior therapy, including CT, IMiD, and PI, whereas four had received CT with either IMiD or PI. In addition, two patients had also received autologous hematopoietic stem cell transplantation. Anemia, bone lesions, an abnormal FLC ratio, a high level of $\beta 2$-microglobulin, and elevated lactate dehydrogenase were commonly observed in most patients at the time of the enrollment. BCMA-positive plasmablasts were observed in all cases $(3.0 \%-81.8 \%)$. Four patients had existing extramedullary lesions.

Among the nine patients, all had HBV infection or previous $\mathrm{HBV}$ infection (table 2). Specifically, we detected $\mathrm{HBs} A g$ positive, $\mathrm{HBeAb}$ positive, and $\mathrm{HBcAb}$ positive in one case; $\mathrm{HBeAb}$ positive and $\mathrm{HBcAb}$ positive in one case; $\mathrm{HBcAb}$ positive in one case; $\mathrm{HBs} A b$ positive, $\mathrm{HBeAb}$ positive, and $\mathrm{HBcAb}$ positive in two cases; and $\mathrm{HBsAb}$ positive and $\mathrm{HBcAb}$ positive in four cases. The HBV DNA level for all patients was below the detection limit $(<100 \mathrm{IU} / \mathrm{mL})$.

\section{Safety}

Prophylaxis with antiviral drugs was recommended for all nine patients with MM. However, patient 6 (HBsAg positive) had been treated with entecavir/lamivudine; patients 2 and 3, who had a resolved HBV infection, had antiviral drug prophylaxis; other patients did not receive antiviral prophylaxis for various reasons after the CAR infusion. The bilirubin, transaminase, and albumin levels were determined after the infusion (figure 2). The median peak total bilirubin (TBIL) level was $14.3 \mu \mathrm{M}$ (range, 6.5-96.8 $\mu \mathrm{M}$; normal range, $0-21 \mu \mathrm{M}$ ), the median peak direct bilirubin (DBIL) level was 5.1 $\mu \mathrm{M}$ (range, 4.1-80.2 $\mu \mathrm{M}$; normal range, $0-5 \mu \mathrm{M})$, and the median peak indirect bilirubin (IBIL) level was 9.2 $\mu \mathrm{M}$ (range, 5.5-16.6 $\mu \mathrm{M}$; normal range, $0-15 \mu \mathrm{M})$. The median peak alanine transaminase (ALT) level was $32 \mathrm{U} / \mathrm{L}$ (range, 10-98 U/L; normal range, 5-40 U/L), and the median peak aspartate transaminase (AST) level was $27 \mathrm{U} / \mathrm{L}$ (range, 11-250 $\mathrm{U} / \mathrm{L}$; normal range, 8-40 U/L). The median low albumin level was $30.7 \mathrm{~g} / \mathrm{L}$ (range, 19.6-40.2 g/L; normal range, 34-48 g/L). Among which, patient 1 had elevated TBIL, DBIL, IBIL, ALT, and AST on days 3-10, up to grade 4 adverse events; patient 5 had elevated TBIL, DBIL, ALT and AST on days 3-10, up to grade 3 adverse events (online supplementary table 2); patients $1,2,4$, 7, and 8 had reduced albumin on days $3-10$. The abnormalities in these patients may be related to CRS or CT. During later follow-up, patient 9 had elevated ALT, AST on days 124-131 (up to grade 1 adverse events), and had elevated AST on days 180-190 (up to grade 2 adverse events, online supplementary figure 1); the other patients had no obvious abnormalities in bilirubin, transaminase, or albumin.

HBV serologies and HBV DNA copy numbers were monitored for all patients after the CAR-T cells infusion. For patient 6 (HBsAg positive), although the HBV DNA replication was below the lower limit of detection after the infusion, the lack of dynamic detection for HBV DNA levels during and after CAR-T cell therapy may be a limitation to this study. Of the patients who had resolved HBV infection, patient 9 was HBsAb and $\mathrm{HBcAb}$ positive before the CAR-T cells infusion, but her HBsAb became negative on day 28 after CAR-T cells infusion, and HBsAg appeared positive on day 180 after CAR-T cells infusion, indicating that HBV reactivation occurred in this patient. She was given entecavir (1 mg/ day) at this time. Two months later antiviral therapy, her HBsAg became negative, HBsAb appeared positive. The HBV DNA level was checked two times before and after the HBsAg changed positive again, and found it was all below the detectable limit (online supplementary table 3). The other patients did not exhibit positivity for HBsAg or HBV DNA replication (table 2). In addition, for the patient 6 (HBsAg positive), the aspartate aminotransferase-to-platelet ratio index (APRI) score was $<0.5$ before and after BCMA CAR-T cell therapy, the FIB-4 score was 1.85 before treatment and 1.10-2.02 after CAR-T cell therapy (online supplementary table 4 ), which showed that CAR-T therapy did not affect the degree of liver fibrosis.

Eight patients had adverse events (table 3 ). The most common events of grade 3 or higher were hematologic toxic effects, including neutropenia, leukopenia, anemia, and thrombopenia; the adverse events may be due to toxicity caused by lymphodepleting CT. Seven (77.78\%) patients had CRS. A higher incidence of CRS was also associated with high cytokine secretion and increased levels of serum CRP and serum ferritin (figure 3A). CRS occurred early after the CAR-T cells infusion, with a median time of onset of 1 day (range, 1-3 days) and a median duration of 4 days (range, 1-10 days). Figure 3B,C shows representative results for patient 1 and patient 5, separately. Other symptoms related to CRS are shown in table 3 . None of the patients had CAR-related encephalopathy syndrome . 
Open access

a

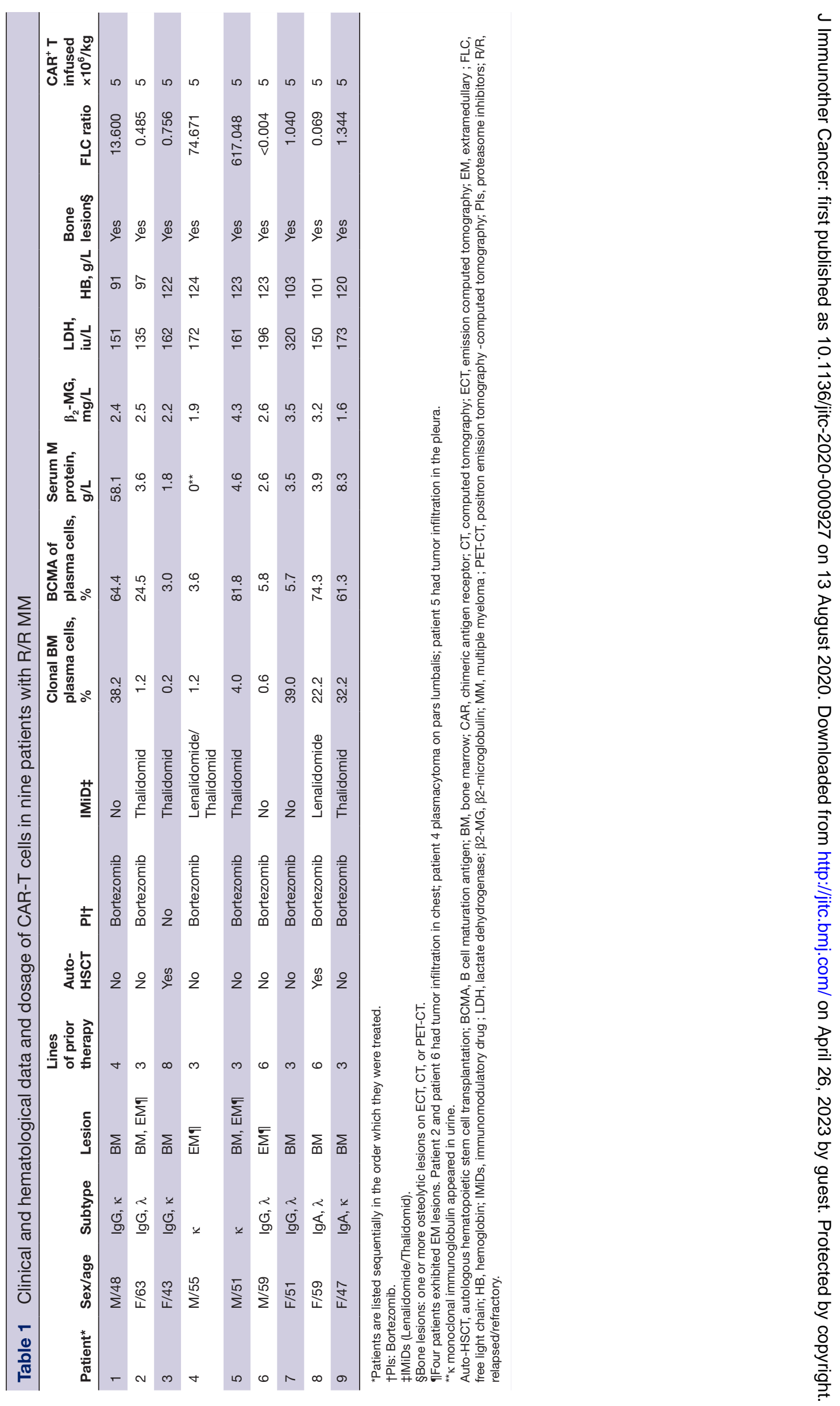

Han L, et al. J Immunother Cancer 2020;8:e000927. doi:10.1136/jitc-2020-000927 


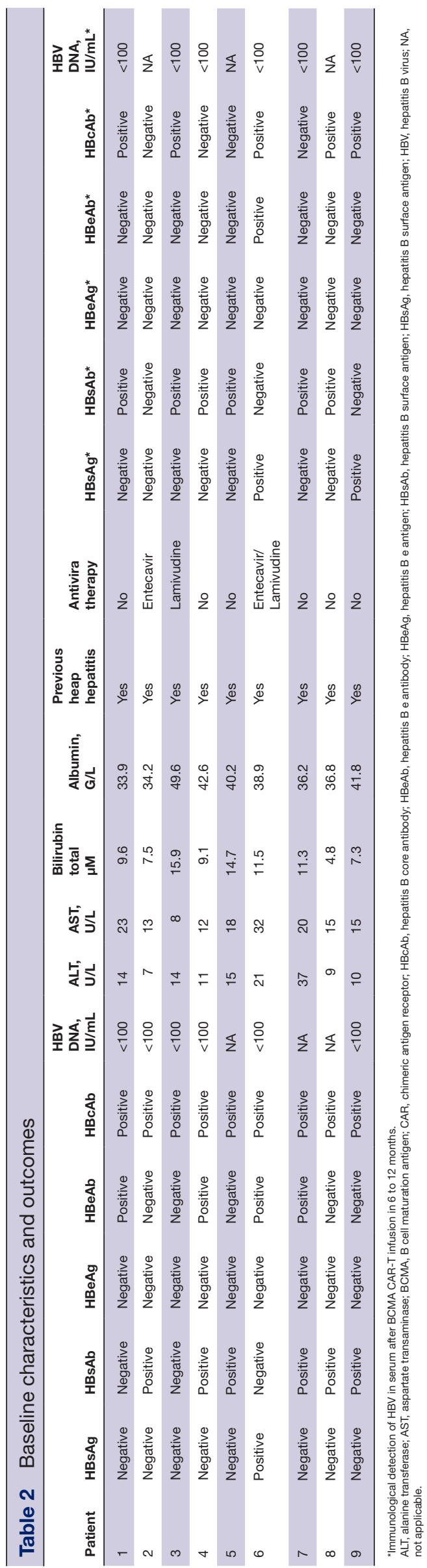

All patients receiving CAR-T cell therapy had low levels of IgG, IgA, and IgM at 1 month, and these low levels lasted for at least 3 months to more than 1 year (figure 3D and online supplementary tables 5-7). All patients received Ig replacement infusion.

\section{Efficacy}

At 1 month after the CAR-T cell therapy, all patients $(100 \%)$ achieved an objective response (figure 4A). During the follow-up period from 3.7 to 12.1 months (median, 9.8 months) for nine patients, as of November 22, 2019, six (66.67\%) patients achieved stringent CR (sCR), two $(22.22 \%)$ patients exhibited very good partial response (PR), and one $(11.11 \%)$ patient exhibited minimal response (figure 4A). Notably, although CR was achieved early in some cases, efficacy improved over time in other patients. Relapse occurred in one patient after sCR. Kaplan-Meier curve analysis showed a PFS rate of 88.89\% (95\% CI, 43\%-98\%) at 12 months (figure 4B); the median PFS was not determined. Because of the small sample size in our study, subgroups could not be analyzed according to tumor BCMA levels, baseline serum results, previous treatment exposure, high-risk cytogenetic profiles, CRS, and CAR-T cells expansion in vivo.

Responses occurred early, and the median time to the first PR or better was 4 weeks (range, 4-10 weeks). Serum $\mathrm{M}$ protein levels declined slowly, leading to a gradual improvement in the response over time in some patients. Compared with the baseline levels, the plasma cells were completely cleared within 4 weeks in most patients, except for patients 7 and 9 (figure 4C); in addition, nearly complete decrease $(>90 \%)$ from the baseline was observed in tumor-associated serum FLC within 4 weeks. Eight patients (excluding patient 3 ) were MRD negative, with an estimated $10^{5}$ nucleated cells at 4 weeks. During follow-up, patient 9 became MRD positive at week 16, whereas all other patients were MRD negative at the posttreatment efficacy evaluation (for which the longest duration was more than 12 months; see figure $4 \mathrm{~A}, \mathrm{C}$ and $\mathrm{D}$ for representative results for patient 5). Tumor responses in sites of extramedullary disease were also observed by 4 weeks, for example, for patient 6 (figure 4E).

The levels of CAR-T cells were easily detected by the CAR DNA copy number, and the results showed high in vivo proliferation (figure 4F). The number of CAR-T cells typically began to increase on the second day and peaked at 5-20 days. The median peak CAR DNA copy number was 512537.14 copies/ $\mu$ g genomic DNA (range: 6846.63$1,716,880.39$ copies/ $\mu g$ genomic DNA). The peak values were not dependent on the initial dose of CAR-T administered (table 1 and figure $4 \mathrm{~F}$ ). In addition, the CAR-T cells expansion in patients 1 and 2 did not appear to be negatively affected by tocilizumab or glucocorticoid use.

\section{DISCUSSION}

This is the first systematic analysis of the safety and efficacy of BCMA CAR-T cell therapy in patients with MM 

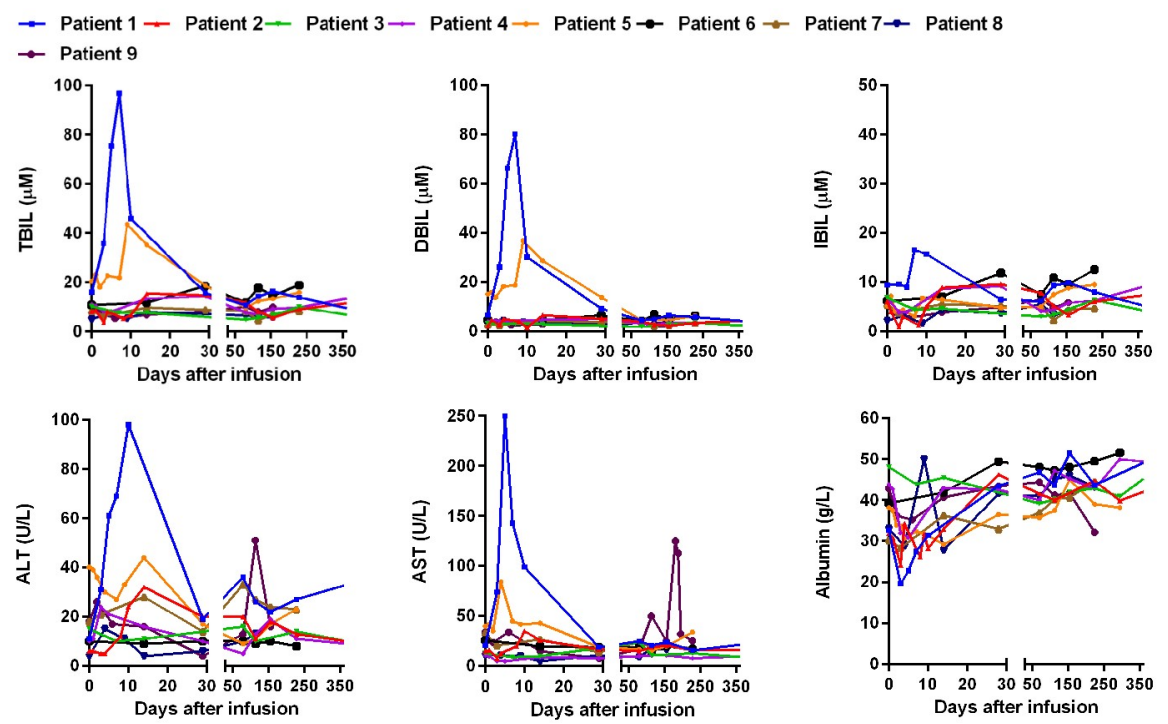

Figure 2 Hepatic function of BCMA CAR-T in MM with chronic HBV infection. Expression of TBIL (normal range, 0 to $21 \mu \mathrm{M}$ ), DBIL (normal range, 0 to $5 \mu \mathrm{M}$ ), IBIL (normal range, 0 to $15 \mu \mathrm{M}$ ), ALT (normal range, 5 to $40 \mathrm{U} / \mathrm{L}$ ), AST(normal range, 8 to $40 \mathrm{U} / \mathrm{L}$ ) and albumin (normal range, 34 to $48 \mathrm{~g} / \mathrm{L}$ ) after CAR-T cells infusion. ALT, alanine transaminase; AST, aspartate transaminase; CAR, chimeric antigen receptor; DBIL, direct bilirubin; IBIL, indirect bilirubin; TBIL, total bilirubin.

and concomitant HBV infection. Our findings showed that BCMA CAR-T cells could be safely used to treat patients with HBV-infected MM, if antiviral drugs were administered during the therapy; the curative effects of CAR-T cells did not seem altered by the infection of HBV.

MM is a hematological malignancy with no cure. Despite advancements in treatment strategies, including IMiD, PI, and monoclonal antibodies, ${ }^{24-26}$ almost all patients with a high-risk cytogenetic profile or treatment-refractory disease eventually relapse. ${ }^{27-29}$ Recently, BCMA CAR-T cells have been shown to have promise in the treatment of MM. ${ }^{19} 2030$ In addition, some reports have shown that the prevalence of HBV infection is high in patients with MM. ${ }^{31}{ }^{32}$ However, to avoid viral reactivation or fulminant hepatitis, clinical trials of CAR-T therapies have generally excluded patients with aggressive MM who are coinfected

\begin{tabular}{|c|c|c|c|c|c|c|}
\hline Patient & $\begin{array}{l}\text { Cytopenia } \\
\text { (AE grading) }\end{array}$ & $\begin{array}{l}\text { CRS } \\
\text { (AE grading) }\end{array}$ & CRES & $\begin{array}{l}\text { CRS } \\
\text { grading }\end{array}$ & $\begin{array}{l}\text { Use of } \\
\text { IL-6R inhibitor }\end{array}$ & $\begin{array}{l}\text { Use of } \\
\text { glucocorticoid }\end{array}$ \\
\hline 1 & 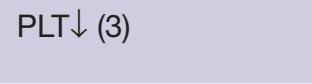 & $\begin{array}{l}\text { Fever (3), Diarrhea (2), Creatinine } \\
\uparrow(1) \text {, Abnormal coagulation (1) }\end{array}$ & No & 2 & Yes & Yes \\
\hline 2 & WBC $\downarrow$ (3) & $\begin{array}{l}\text { Fever (2), Hypoxia (3), Chill (1), } \\
\text { Heart failure (3), hypotension (3) }\end{array}$ & No & 3 & No & Yes \\
\hline 3 & No & No & No & 0 & No & No \\
\hline 4 & $\begin{array}{l}\text { WBC } \downarrow \text { (2), NEU } \downarrow \text { (3), } \\
\text { PLT } \downarrow \text { (3), LYM } \downarrow \text { (2) }\end{array}$ & Fever (2), Hypoglobulinemia (1) & No & 1 & No & No \\
\hline 5 & No & $\begin{array}{l}\text { Fever (1), Bilirubin } \uparrow(1), \text { D-Dimer } \\
\text { (1), LDH } \uparrow(1), \alpha-H B D H \uparrow(1)\end{array}$ & No & 1 & No & No \\
\hline 6 & No & No & No & 0 & No & No \\
\hline 7 & $\begin{array}{l}\text { WBC } \downarrow \text { (4), NEU } \downarrow \text { (4), } \\
\mathrm{PLT} \downarrow \text { (4), LYM } \downarrow \text { (3), } \\
\text { MON } \downarrow \text { (2) }\end{array}$ & Fever (2) & No & 1 & No & No \\
\hline 8 & $\begin{array}{l}\text { WBC } \downarrow \text { (4), NEU } \downarrow \text { (4), } \\
\text { PLT } \downarrow \text { (4), LYM } \downarrow \text { (4) }\end{array}$ & Fever (1), NT-BNP (1) & No & 2 & No & No \\
\hline 9 & LYM $\downarrow$ (4) & Fever (2) & No & 1 & No & No \\
\hline
\end{tabular}

$\downarrow$ indicates decrease ; $\uparrow$ indicates increase.

The grading of AE was according to the CTCAE V.4.03.

AEs, adverse events; ; CRES, CAR-related encephalopathy syndrome; CRS, cytokine release syndrome; $\alpha-\mathrm{HBDH}, \alpha$ hydroxybutyrate dehydrogenase; LDH, lactate dehydrogenase; LYM, lymphocyte; NEU, neutrophil count; NT-BNP, N-terminal B-type natriuretic peptide; PLT, platelet count; WBC, white blood cell. 
A

C

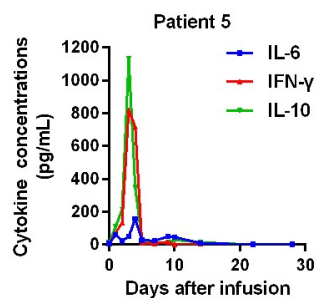

B
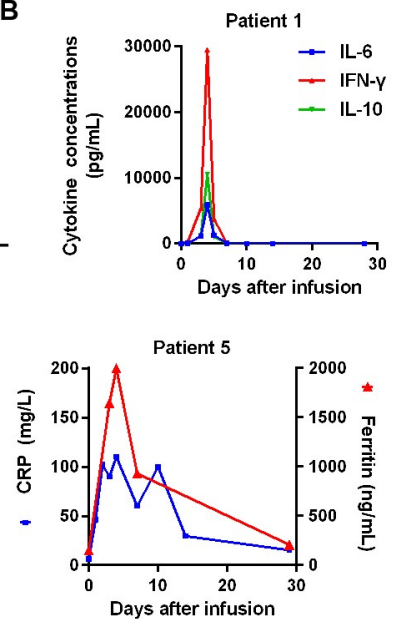

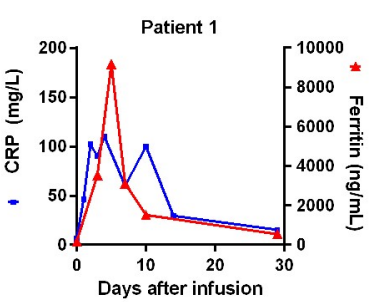

D

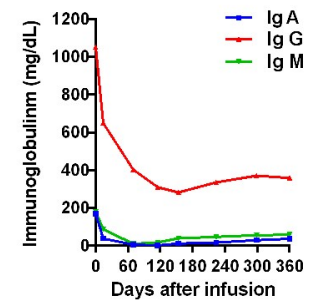

Figure 3 Adverse events of BCMA CAR-T cell therapy in R/R MM. (A) Fold change (peak concentration compared with baseline) of serum cytokines (IL-6, IL-10, and interferon (IFN)- $\gamma$ ), ferritin, and CRP after CAR-T cells infusion in patients with MM. $(B, C)$ Changes in the levels of cytokines, serum ferritin, and serum CRP in representative cases (patient 1 and patient 5) after CAR-T cells infusion. (D) Changes in serum immunoglobulin (lg)A (normal range, 90 to $450 \mathrm{mg} / \mathrm{dL}$ ), IgG (normal range, 800 to $1800 \mathrm{mg} / \mathrm{dL}$ ), and IgM (normal range, 60 to $280 \mathrm{mg} / \mathrm{dL}$ ) levels after CAR-T cell therapy. BCMA, B cell maturation antigen; CAR, chimeric antigen receptor; CRP, C-reactive protein; MM, multiple myeloma; R/R, refractory/relapsed.

with HBV. Accordingly, it was unclear whether CAR-T therapy could be effectively applied to HBV-infected patients with MM.

Patients who were HBsAg positive with malignant tumors may experience $\mathrm{HBV}$ reactivation during anticancer treatment, such as CT. ${ }^{11}$ Delayed HBV reactivation has occurred months after anticancer therapy by rituximab because of immunosuppression with progressive B cell depletion. ${ }^{33-35}$ CAR-T cell therapy may be more likely to cause $\mathrm{HBV}$ reactivation because it is more complex than common methods of MM therapy. High-intensity CT with fludarabine and cyclophosphamide is required before CAR-T cells infusion, and serious CRS may occur after the infusion; any of these may cause $\mathrm{HBV}$ reactivation. However, Strati et al were the first to report the safe treatment of a patient who was HBsAg positive with diffuse large B cell lymphoma (DLBCL) with CD19 CAR-T cells. ${ }^{36}$ This patient remained on tenofovir prescribed at the same dose, before and after the CAR-T cells infusion. The patient had grade 3-4 CRS and achieved CR, with a PFS time of 8 months after therapy, until the follow-up cut-off, and with no reactivation of $\mathrm{HBV}$ or significant increase of ALT and bilirubin. Similar to the report by Strati et $a l,{ }^{36}$ we also treated an patient (patient 6) with HBsAg positive MM with BCMA CAR-T cells under the protection of entecavir. This patient achieved PR and remained at the sCR state after the 9.8 month observation. Most importantly, this patient did not experience HBV reactivation. These results indicate that CAR-T cells can safely and effectively be used to treat patients who were HBV-infected under the protection of anti-HBV drugs, even patients who were HBsAg positive. Wei $\mathrm{J} e t a l^{37}$ reported that a patient who had HBsAg-positive DLBCL died of $\mathrm{HBV}$ reactivation during CAR-T cell therapy because the patient discontinued antiviral drugs, suggesting the importance of concomitant antiviral and CAR-T cell therapy in patients with cancer who were $\mathrm{HBV}$-infected .

Although $\mathrm{HBV}$ reactivation has been reported in patients with resolved HBV infection during the application of new anticancer drugs, ${ }^{48} 39$ CAR-T cell therapy in patients with MM who have resolved $\mathrm{HBV}$ may be safer than that in patients who were HBsAg positive. In our present study, of the eight patients with MM who have resolved $\mathrm{HBV}$ infection, two patients administered prophylactic anti-HBV drugs did not exhibit HBV reactivation; of the six patients who did not use prophylactic antiviral drugs, five did not exhibit HBV reactivation, only one showed recurrence of $\mathrm{HBsAg}$ without detection of HBV DNA or damage to liver function. However, among the six patients who did not use prophylactic antiviral drugs, two patients had elevated liver function within the early 2 weeks. Although the abnormal liver function might be associated with CRS or CT, hepatitis B serology and HBV DNA levels should be checked at this time to distinguish if this was caused by $\mathrm{HBV}$ reactivation. The lack of hepatitis B serology and HBV DNA in this study is a limitation for the diagnoses of the two patients. These above results suggest that CAR-T cell therapy is safe for patients with MM who have resolved HBV. While prophylaxis is recommended for all patients, for those who cannot or do not take prophylaxis, CAR-T cell therapy may still be feasible with close laboratory monitoring of HBV serologies and HBV DNA, and prompt initiation of antiviral therapy if turning positive. Strati $e t a l^{36}$ reported that a patient with DLBCL who had resolved HBV exhibited HBV DNA reactivation after 3 months because of self-discontinued antiviral prophylaxis 13 months after infusion; this indicates that anti-HBV therapy should be administered until the patient serum Ig has recovered. In addition, in the present study, of the six patients who 


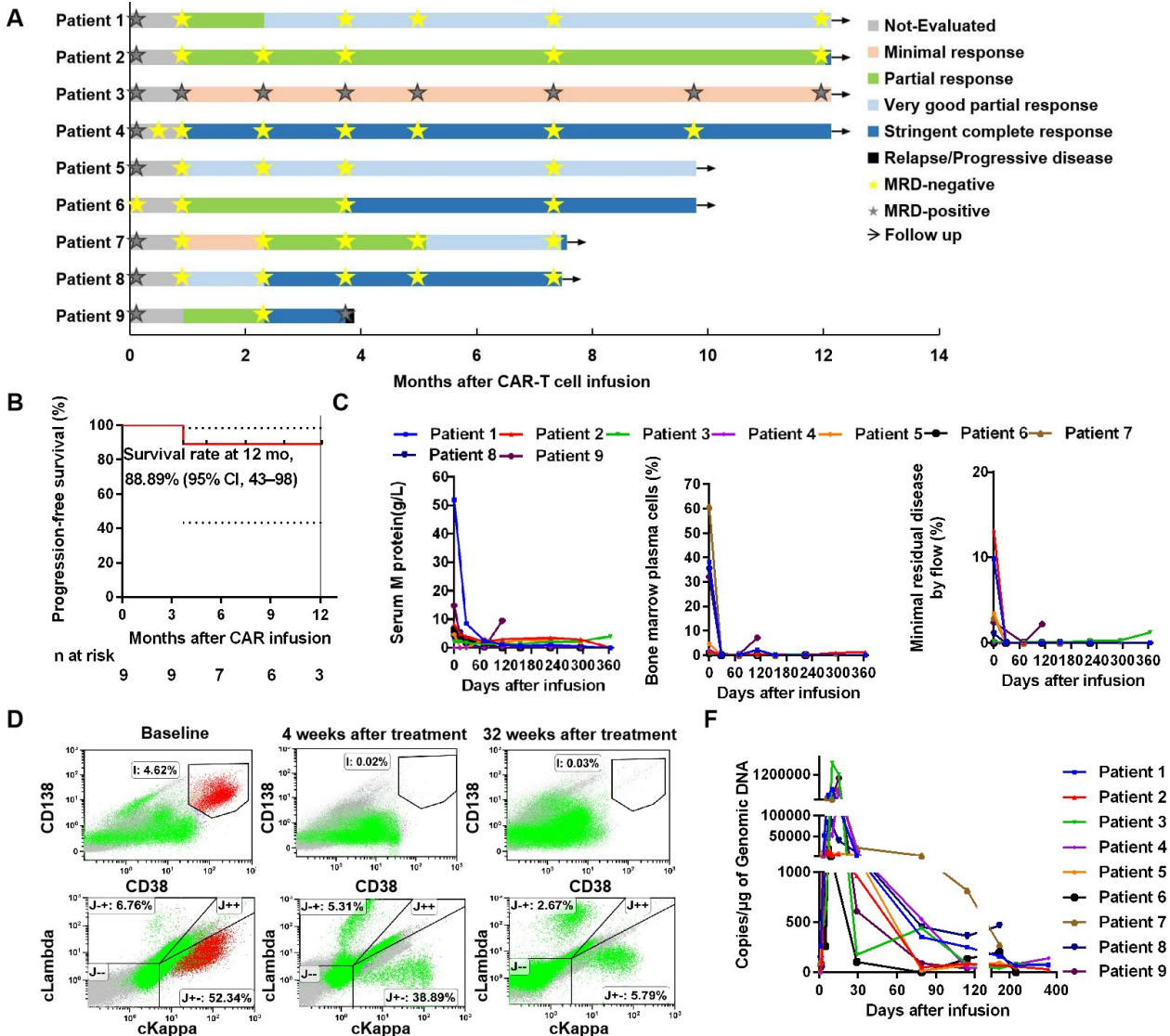

E

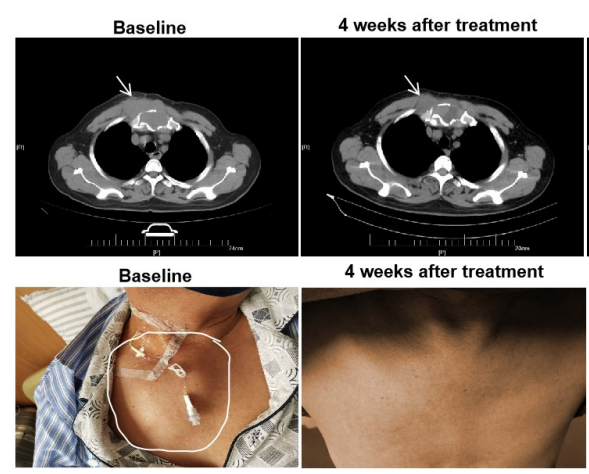

22 weeks after treatment

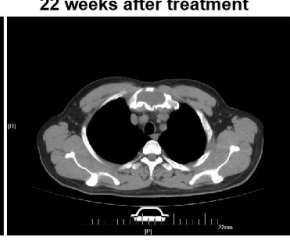

Figure 4 Clinical activity after BCMA CAR-T cells infusion in patients. (A) Best responses among the nine patients. All responses were confirmed and assessed according to the International Myeloma Working Group uniform response criteria for MM. MRD denotes minimal residual disease based on flow cytometry analysis. (B) Prognosis of patients with MM after the BCMA CAR-T cells infusion. The PFS was calculated by the Kaplan-Meier method. (C) Changes in plasma M-protein concentrations (left), plasma cells in the bone marrow by bone marrow cytological examination (middle), and MRD by flow cytometry (right) after BCMA CAR-T cell therapy. (D) Flow cytometry analysis of bone marrow plasma cells in a representative case (patient 5) after BCMA CAR-T cells treatment. No abnormal plasma cells and light chain-related tumor cells were found on reexamination. (E) Response of extramedullary infiltration lesions in a representative case (patient 6) after CAR-T cells infusion. The mass in the chest was reduced in size at 4 weeks and then disappeared at 22 weeks, as demonstrated by CT and surface observation. white arrows indicate sites of tumor lesions. (F) Gene-modified T cells in peripheral blood were assessed by quantitative real-time PCR. The horizontal line at 5 copies/mg DNA represents the lower limit of quantification of this assay. Data at the first time point were obtained before infusion of BCMA CAR-T cells. BCMA, B cell maturation antigen; CAR, chimeric antigen receptor; PFS, progression-free survival.

did not use prophylactic antiviral drugs, only one had evidence for reactivation; this may be associated with patients receiving intravenous immunoglobulin replacement, which contributed to the low re-activation rate.

Another risk of CAR-T cell therapy is that the HBV infection aggravates CRS. Fortunately, of the nine patients who were HBV-infected in our study, no patient died of CRS after CAR-T cell therapy. Six patients experienced grade 1-2 CRS, and one patient experienced grade 3 CRS, all of whom were controlled by symptomatic support and IL-6 therapy. These results indicated that the HBV infection did not aggravate CRS; however, further studies 
are needed to clarify the risk of CRS in the presence of chronic HBV infection. Importantly, the HBV infection did not seem to affect the effectiveness of the CAR-T cell therapy; of the nine patients with MM treated, the best ORR was $100 \%$, and the PFS rate at 12 months was $88.89 \%$, which were comparable with the curative effects of other clinical trials. ${ }^{19} 20$

In conclusion, for patients with MM with resolved HBV infection, BCMA CAR-T cell therapy can be administered safely and the clinical efficacy dose not seem to be affected. However, strictly monitor the HBV infection and concurrently administer antiviral therapy are recommended to prevent the reactivation of HBV. Because of the small number of patients and the retrospective nature of this study, additional clinical research is needed to clarify the safety and efficacy of this approach.

Acknowledgements The authors thank the patients who participated in this clinical trial. The authors thank The Shenzhen Pregene Biopharma Company, Ltd for kindly providing the viral fluid for chimeric antigen receptor T cells preparation. The authors thank the medical staff of the Department of Immunology, and Department of Hematology, Affiliated Cancer Hospital of Zhengzhou University and Henan Cancer Hospital who participated in this clinical trial.

Contributors LH and QG provided and interpreted data. LH, QG, Y-PS, JZ, K-SZ, and J-SZ provided design input and analyzed the data. QG conceived and designed the study and interpreted data. LH drafted the final manuscript. All authors reviewed and approved the final manuscript.

Funding This study was supported by grants from Henan Medical Science and Technique Foundation (grant number 2018020484; SBGJ2018085); Henan Provincial Scientific and Technological Project (grant number 162300410095); Natural Science Foundation of Henan (grant number 182300410344); Henan Province Industry-University-Research Cooperation Project (grant number 182107000027).

\section{Competing interests None declared.}

Patient consent for publication Not required.

Ethics approval The study was approved by the Ethics Committee of the Affiliated Cancer Hospital of Zhengzhou University. Informed consent was obtained from all patients.

Provenance and peer review Not commissioned; externally peer reviewed.

Data availability statement Data are available upon reasonable request. The datasets used and/or analyzed during the current study are available from the corresponding author on reasonable request.

Open access This is an open access article distributed in accordance with the Creative Commons Attribution 4.0 Unported (CC BY 4.0) license, which permits others to copy, redistribute, remix, transform and build upon this work for any purpose, provided the original work is properly cited, a link to the licence is given, and indication of whether changes were made. See https://creativecommons.org/ licenses/by/4.0/.

\section{ORCID iD}

Quanli Gao http://orcid.org/0000-0002-6314-0276

\section{REFERENCES}

1 Schweitzer A, Horn J, Mikolajczyk RT, et al. Estimations of worldwide prevalence of chronic hepatitis $B$ virus infection: a systematic review of data published between 1965 and 2013. Lancet 2015;386:1546-55.

2 Hoofnagle JH. Reactivation of hepatitis B. Hepatology 2009;49:S156-65

3 Yeo W, Chan PK, Zhong S, et al. Frequency of hepatitis B virus reactivation in cancer patients undergoing cytotoxic chemotherapy: a prospective study of 626 patients with identification of risk factors. $J$ Med Virol 2000;62:299-307.
4 Dervite I, Hober D, Morel P. Acute hepatitis B in a patient with antibodies to hepatitis $B$ surface antigen who was receiving rituximab. N Engl J Med 2001;344:68-9.

5 Hui C-K, Cheung WWW, Zhang H-Y, et al. Kinetics and risk of de novo hepatitis $\mathrm{B}$ infection in $\mathrm{HBsAg}$-negative patients undergoing cytotoxic chemotherapy. Gastroenterology 2006;131:59-68.

6 Cheng A-L, Hsiung CA, Su I-J, et al. Steroid-Free chemotherapy decreases risk of hepatitis $B$ virus (HBV) reactivation in HBV-carriers with lymphoma. Hepatology 2003;37:1320-8.

7 Totani $\mathrm{H}$, Kusumoto S, Ishida T, et al. Reactivation of hepatitis B virus (HBV) infection in adult T-cell leukemia-lymphoma patients with resolved HBV infection following systemic chemotherapy. Int $J$ Hematol 2015;101:398-404

8 Yuki N, Nagaoka T, Yamashiro M, et al. Long term histologic and virologic outcomes of acute self-limited hepatitis B. Hepatology 2003;37:1172-9.

9 Rehermann B, Ferrari C, Pasquinelli C, et al. The hepatitis B virus persists for decades after patients' recovery from acute viral hepatitis despite active maintenance of a cytotoxic T-lymphocyte response. Nat Med 1996:2:1104-8.

10 Locasciulli A, Bruno B, Alessandrino EP, et al. Hepatitis reactivation and liver failure in haemopoietic stem cell transplants for hepatitis $B$ virus (HBV)/hepatitis C virus (HCV) positive recipients: a retrospective study by the Italian group for blood and marrow transplantation. Bone Marrow Transplant 2003;31:295-300.

$11 \mathrm{Kim}$ HY, Kim W. Chemotherapy-related reactivation of hepatitis B infection: updates in 2013. World J Gastroenterol 2014;20:14581-8.

12 Idilman R, Arat M. Evaluation and management of hepatitis $B$ virus infection in hematopoietic stem cell transplantation: before and after transplantation. Expert Rev Anti Infect Ther 2011;9:641-52.

13 Yeo W, Chan TC, Leung NWY, et al. Hepatitis B virus reactivation in lymphoma patients with prior resolved hepatitis B undergoing anticancer therapy with or without rituximab. J Clin Oncol 2009;27:605-11.

14 Makita S, Yoshimura K, Tobinai K. Clinical development of anti-CD19 chimeric antigen receptor T-cell therapy for B-cell non-Hodgkin lymphoma. Cancer Sci 2017;108:1109-18.

15 Neelapu SS, Locke FL, Go WY. Car T-cell therapy in large B-cell lymphoma. N Engl J Med 2018;378:1065.

16 Mikkilineni L, Kochenderfer JN. Chimeric antigen receptor T-cell therapies for multiple myeloma. Blood 2017;130:2594-602.

17 Ali SA, Shi V, Maric I, et al. T cells expressing an anti-B-cell maturation antigen chimeric antigen receptor cause remissions of multiple myeloma. Blood 2016;128:1688-700.

18 Brudno JN, Maric I, Hartman SD, et al. T cells genetically modified to express an anti-B-cell maturation antigen chimeric antigen receptor cause remissions of poor-prognosis relapsed multiple myeloma. $J$ Clin Oncol 2018;36:2267-80.

$19 \mathrm{Xu} \mathrm{J}$, Chen L-J, Yang S-S, et al. Exploratory trial of a biepitopic CAR T-targeting $B$ cell maturation antigen in relapsed/refractory multiple myeloma. Proc Natl Acad Sci U S A 2019;116:9543-51.

20 Raje N, Berdeja J, Lin Y, et al. Anti-BCMA CAR T-cell therapy bb2121 in relapsed or refractory multiple myeloma. N Engl J Med 2019;380:1726-37.

21 Ataca Atilla P, Yalçıner M, Atilla E, et al. Hepatitis B reactivation rate and fate among multiple myeloma patients receiving regimens containing lenalidomide and/or bortezomib. Turk $J$ Haematol 2019;36:266-73.

22 Lee DW, Gardner R, Porter DL, et al. Current concepts in the diagnosis and management of cytokine release syndrome. Blood 2014;124:188-95.

23 Kumar S, Paiva B, Anderson KC, et al. International myeloma Working group consensus criteria for response and minimal residual disease assessment in multiple myeloma. Lancet Oncol 2016;17:e328-46.

24 Palumbo A, Anderson K. Multiple myeloma. N Engl J Med 2011;364:1046-60.

25 Kumar SK, Dispenzieri A, Lacy MQ, et al. Continued improvement in survival in multiple myeloma: changes in early mortality and outcomes in older patients. Leukemia 2014;28:1122-8.

26 Chim CS, Kumar SK, Orlowski RZ, et al. Management of relapsed and refractory multiple myeloma: novel agents, antibodies, immunotherapies and beyond. Leukemia 2018;32:252-62.

27 Kumar SK, Lee JH, Lahuerta JJ, et al. Risk of progression and survival in multiple myeloma relapsing after therapy with IMiDs and bortezomib: a multicenter international myeloma Working Group study. Leukemia 2012;26:149-57.

28 Sonneveld P. Management of multiple myeloma in the relapsed/ refractory patient. Hematology Am Soc Hematol Educ Program 2017;2017:508-17. 
29 Nijhof IS, van de Donk NWCJ, Zweegman S, et al. Current and new therapeutic strategies for relapsed and refractory multiple myeloma: an update. Drugs 2018;78:19-37.

30 Zhao W-H, Liu J, Wang B-Y, et al. A phase 1, open-label study of LCAR-B38M, a chimeric antigen receptor $\mathrm{T}$ cell therapy directed against $B$ cell maturation antigen, in patients with relapsed or refractory multiple myeloma. J Hematol Oncol 2018;11:141.

31 Huang B, Li J, Zhou Z, et al. High prevalence of hepatitis B virus infection in multiple myeloma. Leuk Lymphoma 2012;53:270-4.

$32 \mathrm{He} \mathrm{L-L,} \mathrm{Ke} \mathrm{Y.} \mathrm{[Monitoring} \mathrm{of} \mathrm{HBsAg} \mathrm{and} \mathrm{Risk} \mathrm{of} \mathrm{HBV} \mathrm{Infection} \mathrm{in}$ Patients with Multiple Myeloma]. Zhongguo Shi Yan Xue Ye Xue Za Zhi 2017;25:1718-21.

33 Sarrecchia C, Cappelli A, Aiello P. HBV reactivation with fatal fulminating hepatitis during rituximab treatment in a subject negative for $\mathrm{HBsAg}$ and positive for $\mathrm{HBsAb}$ and $\mathrm{HBcAb}$. J Infect Chemother 2005;11:189-91.

34 Law JK, Ho JK, Hoskins PJ, et al. Fatal reactivation of hepatitis B post-chemotherapy for lymphoma in a hepatitis B surface antigennegative, hepatitis $B$ core antibody-positive patient: potential implications for future prophylaxis recommendations. Leuk Lymphoma 2005;46:1085-9.

35 Niscola P, Del Principe MI, Maurillo L, et al. Fulminant B hepatitis in a surface antigen-negative patient with B-cell chronic lymphocytic leukaemia after rituximab therapy. Leukemia 2005;19:1840-1.

36 Strati P, Nastoupil LJ, Fayad LE, et al. Safety of car T-cell therapy in patients with B-cell lymphoma and chronic hepatitis B or $\mathrm{C}$ virus infection. Blood 2019;133:2800-2.

37 Wei J, Zhu X, Mao X, et al. Severe early hepatitis B reactivation in a patient receiving anti-CD19 and anti-CD22 CAR T cells for the treatment of diffuse large B-cell lymphoma. J Immunother Cancer 2019;7:315

38 Tsutsumi Y, Tanaka J, Kawamura T, et al. Possible efficacy of lamivudine treatment to prevent hepatitis $B$ virus reactivation due to rituximab therapy in a patient with non-Hodgkin's lymphoma. Ann Hematol 2004;83:58-60.

39 Westhoff TH, Jochimsen F, Schmittel A, et al. Fatal hepatitis B virus reactivation by an escape mutant following rituximab therapy. Blood 2003;102:1930. 